\title{
Breathers in a system with helicity and dipole interaction
}

\author{
B. Sánchez-Rey, J. F. R. Archilla, and F. Palmero \\ Departamento de Física Aplicada I, Universidad de Sevilla, Avenida Reina Mercedes s/n, 41012-Sevilla, Spain \\ F. R. Romero \\ Facultad de Física, Universidad de Sevilla, Avenida Reina Mercedes s/n, 41012-Sevilla, Spain \\ (Received 31 January 2002; published 3 July 2002)
}

\begin{abstract}
Recent papers that have studied variants of the Peyrard-Bishop model for DNA, have taken into account the long range interaction due to the dipole moments of the hydrogen bonds between base pairs. In these models the helicity of the double strand is not considered. In this paper we have performed an analysis of the influence of the helicity on the properties of static and moving breathers in a Klein-Gordon chain with dipole-dipole interaction. It has been found that the helicity enlarges the range of existence and stability of static breathers, although this effect is small for a typical helical structure of DNA. However, the effect of the orientation of the dipole moments is considerably higher with transcendental consequences for the existence of mobile breathers.
\end{abstract}

DOI: 10.1103/PhysRevE.66.017601 PACS number(s): 63.20.Pw, 63.20.Ry, 63.50.+x, 87.10.+e

\section{INTRODUCTION}

A great deal of attention has been paid to the interplay between geometry and nonlinearity in locating problems in recent years. The relationship between geometry and nonlinearity has an important role in the functions of some biomolecules, such as DNA, where the localization of energy has been put forward as a precursory mechanism of the transcription bubble [1], and moving localized excitations as a method of transporting information along the double strand $[2]$.

The fact that hydrogen bonds that link each pair of bases in DNA have a finite dipole moment, has brought about the introduction of models [3-6] with long range dipole-dipole interaction. Apart from its theoretical interest, this interaction becomes relevant when the secondary structure of DNA is considered. The shape of the molecule can influence the localization and transport properties of energy, which is thought to play a biological function [7]. Some of these models [3] study the effects of the curvature in a chain of nonlinear oscillators using the discrete nonlinear Schrödinger equation. Other models consider Klein-Gordon systems to study kinks [4], breathers in curved chains [5] or breathers with two competing interactions [6]. However, all these models with long range interaction fail to take into account the peculiar helicoidal structure of the DNA chain, although this has been considered in some models [8] without the dipole interaction.

In this paper, we study the effect of helicity on the properties of breathers in a Klein-Gordon model with dipoledipole interaction. These periodic nonlinear localized oscillations in discrete systems are very localized excitations that appear as a consequence of the nonlinearity and discreteness of the system [9]. They are specially suitable for biomolecules when considering excitations that involve a few units, that is, far from the continuous limit. They can be static but, under certain conditions, also move and transport energy along the system [10].

We have found that the introduction of helicity enhances the stability of static breathers, although this effect is rela- tively small for the typical helicoidal structure of the DNA. On the other hand, the profile of the static breathers and the properties of moving ones are strongly dependent on the relative orientation between the dipole moments.

\section{THE MODEL}

The model is inspired by the primary structure of DNA, with dipole moments perpendicular to the helix axis, and where the stretching of the hydrogen bonds within base pairs is described as a variation of the dipole moments. More detailed justification of the model can be found in [6].

We denote $\phi_{n}$ the angle of the $n$ dipole with respect to a reference axis perpendicular to the helix axis. Then, the angle between the nearest neighboring dipoles is $\theta_{t w}=\phi_{n}$ $-\phi_{n-1}$. We have considered this neighboring angle constant along the chain, and it will be called the twisting angle. Thus, $\phi_{n+m}-\phi_{n}=m \theta_{t w}$, and, therefore, $2 \pi / \theta_{t w}$ dipoles are needed to complete a turn of screw. In DNA, for example, the twisting angle is $36^{\circ}$ and a turn of screw requires ten base pairs. Figure 1 shows a sketch of the model, where it can be appreciated that the system of dipoles have a helicoidal structure.

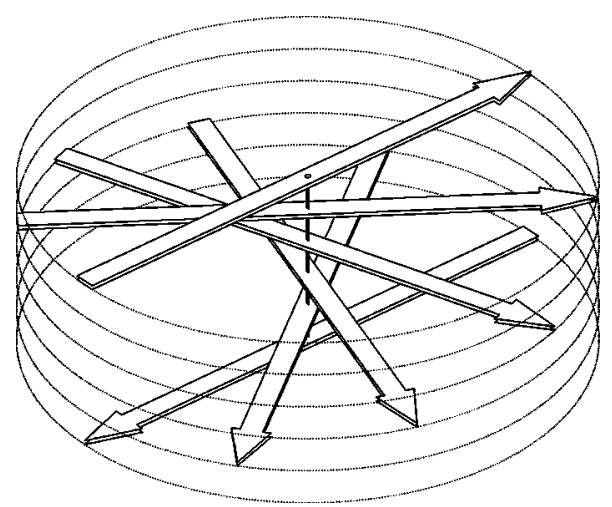

FIG. 1. Sketch of the model at equilibrium. The arrows represent the dipoles moments, perpendicular to the helix axis. 
In the appropriate dimensionless variables, the Hamiltonian of our system becomes

$$
\begin{aligned}
H= & \sum_{n=1}^{N}\left(\frac{1}{2} \dot{u}_{n}^{2}+V\left(u_{n}\right)+\frac{1}{2} J \sum_{m=n-N / 2}^{n+N / 2} \frac{u_{n} u_{m}}{|n-m|^{3}}\right. \\
& \left.\times \cos \left[\theta_{t w}(n-m)\right]\right),
\end{aligned}
$$

where $N$ is the number of variables. The variables $\left\{u_{n}\right\}_{n=1}^{N}$, where $u_{n \pm N}=u_{n}$, represent, in the context of the PeyrardBishop model for DNA [1], the transversal displacements of the two complementary nucleotides in the $n$th pair with respect to the molecular axis. In our model, they describe the stretching of the dipoles with respect to their equilibrium lengths. $V\left(u_{n}\right)$ is the on-site potential, which, in DNA models, describes the hydrogen bonds linking the two bases, and the parameter $J$ measures the strength of the long range dipole-dipole interaction. We have chosen the on-site potential as the Morse potential, given by

$$
V\left(u_{n}\right)=\frac{1}{2}\left(e^{-u_{n}-1}\right)^{2} .
$$

The reason for this, is that it is a suitable potential for representing chemical bonds, being asymmetric, with a hard part, modeling the repulsion between atoms or molecules, and a soft part that becomes flat, modeling the breakage of the bond.

The dynamical equations become

$$
\ddot{u}_{n}+V^{\prime}\left(u_{n}\right)+J \sum_{m=n-N / 2}^{n+N / 2} \frac{\cos \left[\theta_{t w}(n-m)\right]}{|n-m|^{3}} u_{m}=0
$$

where $n=1, \ldots, N$. To study the linear modes of the system we replace $V^{\prime}\left(u_{n}\right)$ in Eq. (3) with the linear term $u_{n}$, which implies that the time has been scaled so that the linear frequency $\omega_{0}=1$. Considering solutions of the form $u_{n}$ $=e^{i q n-i w t}$ the following dispersion relation is obtained:

$$
w_{k}=\sqrt{1+2 J \sum_{m=1}^{N / 2} \frac{\cos \left(m \theta_{t w}\right)}{m^{3}} \cos \left(m q_{k}\right)} \text {, }
$$

where $q_{k}=2 \pi k / N$, with $k=1, \ldots, N$ due to the periodic boundary conditions.

The variation of the phonon band with the helicity is shown in Fig. 2, where the frequencies of the linear modes are represented as a function of the twisting angle, $\theta_{t w}$, for a fixed value of the coupling parameter $J=0.1$. The effect of the twisting is a narrowing of the phonon band, which will enhance the range of existence and stability of the breathers. This has been confirmed numerically.

\section{BREATHER EXISTENCE AND STABILITY}

We have studied the existence and stability of breathers in this model using the standard numerical methods described in Ref. [11].

The Morse potential is a soft potential with the conse-

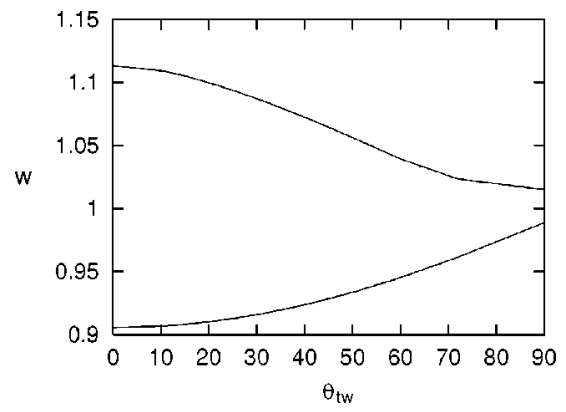

FIG. 2. Effect of helicity on the breadth of the linear spectrum. The curves represent the lower and higher limits of the phonon band as a function of the twisting angle in degrees for fixed coupling parameter $J=0.1 . w$ is in dimensionless units.

quence that the frequency of a breather has to be lower than the linear frequency $\omega_{0}=1$. Thus, we have chosen, $\omega_{\mathrm{b}}$ $=0.8$ so that the nonlinear effect will be significant but on the other hand not overtly strong, as the nonlinearities in DNA are thought to be weak.

First of all, the helicity influences the breather profile. As is shown in Fig. 3, for a fixed value of the coupling parameter $J$, the increase of the twisting angle produces a transition from a zigzag profile (the nearest neighbor oscillating in antiphase) to a bell profile (all dipoles oscillating in phase). This effect follows from the spatial profile of the phonon state with the lowest frequency since the breather frequency is below the phonon band, and all the higher harmonics are way too high to be relevant. For $\theta_{t w}<\pi / 2$ the interaction is effectively "antiferromagnetic," which leads to staggered phonons at the lower band edges. In the same way for $\theta_{t w}$ $>\pi / 2$ a "ferromagnetic" interaction is present, which leads to a nonstaggered phonon at the lower band edge. The breather bifurcates from the lower band edge phonons and thus retains the property of the phonon structure. For $\theta_{t w}$ $=\pi / 2$ the system separates into two noninteracting sublattices: even and odd sites. As a result, in this case, the nearest neighbors are at rest and the odd site sublattice remains unexcited.

One-site breathers are stable at low coupling as was proved by Aubry [12]. For any value of the twisting angle $\theta_{t w}<90^{\circ}$ they can be continued from the anticontinuous limit till $\omega_{\mathrm{b}}$ enters the phonon band. Just before the breather disappears, it becomes unstable due to the occurrence of a

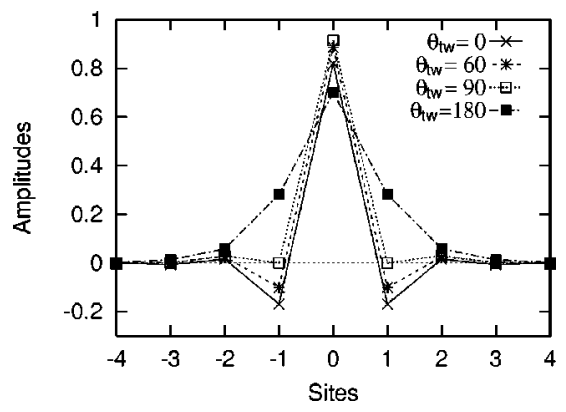

FIG. 3. Profiles of the one-site breather when twisting is increased for fixed coupling $J=0.1$ and Morse potential. Dimensionless units. 


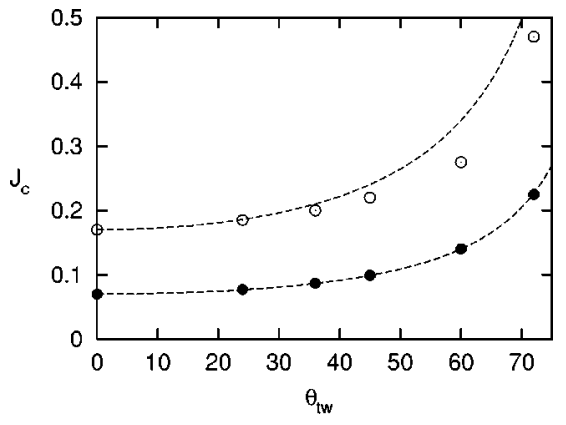

FIG. 4. Range of stability of the one-site (circles) and two-site (full circles) breathers as a function of the twisting angle in degrees. $J_{c}$ is the maximum value of the coupling parameter for which the breather is stable. The dashed lines represent the values calculated within the NNI approximation. $J_{c}$ is in dimensionless units.

harmonic bifurcation in the evolution of the Floquet eigenvalues. The increase of the twisting enhances the stability as is shown in Fig. 4 (circles). This can be understood if we consider only the nearest neighbor interaction (NNI). Then the influence of helicity on the stability of the breathers could be described by an effective coupling $J_{e f f}=J \cos \theta_{t w}$. The one-site breather without twisting loses its stability for a coupling value of $J_{c}^{0}$. With twisting and only NNI this would occur for $J_{c}=J_{c}^{0} / \cos \theta_{t w}$ (dash lines in Fig. 4), which concurs with the numerical results.

The two-site breather, which consist of two neighboring oscillators excited in phase, is also stable at low coupling. This can be understood in terms of Aubry's band theory [12]. When coupling is increased, a bubble of instability appears due to Krein crunches between the phonon band eigenvalues and a localized eigenvalue of the Floquet operator. If we continue increasing the coupling the double breather definitely becomes unstable due to the occurrence of a subharmonic bifurcation. Again, the effect of the twisting is to enlarge the range of stability toward higher values of the coupling parameter (full circles in Fig. 4). This suggests that twisting might be a way to control the stability of the breathers in real systems.

We have not considered the two-site breather in antiphase because it coincides with the one-site breather with zigzag profile, i.e., the Newton method converges to the same solution if we start at the anticontinuous limit with one nonlinear oscillator or with two nearest neighbor oscillators in antiphase.

A rather different situation is the one with $\theta_{t w}>90^{\circ}$. First, the one-site breather is always stable until it disappears. Second, the two-site breather is unstable at low coupling but becomes stable just before its extinction. This behavior has important consequences for the mobility of these breathers as shown in the following section.

For the sake of thoroughness, we have also studied the effect of twisting with a hard $\phi^{4}$ potential $V\left(u_{n}\right)=u_{n}^{2}$ $+1 / 4 u_{n}^{4}$, and a breather frequency $\omega_{\mathrm{b}}=1.2$. Qualitatively the results are similar except for the fact that breathers with $\theta_{t w}<90^{\circ}$ and breathers with $\theta_{t w}>90^{\circ}$ exchange their properties.

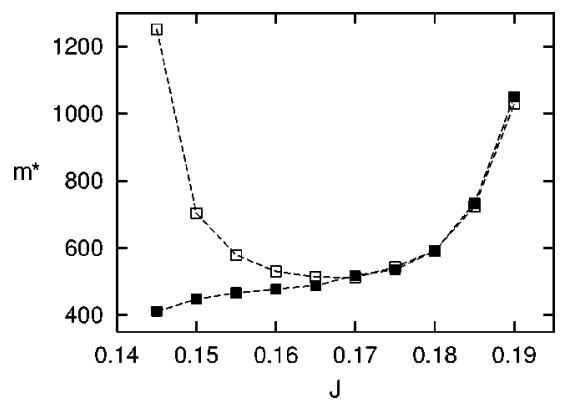

FIG. 5. Dependence of the effective mass of the mobile breathers with the coupling parameter for $\theta_{t w}=180^{\circ}$. The blank squares correspond to mobile breathers obtained from static one-site breathers. Full squares are obtained from static two-site breathers.

\section{MOBILE BREATHERS}

Static breathers under certain conditions can be moved. The standard method to move a breather consists in perturbing its velocity with spatially antisymmetric vector, called the marginal mode [10]. Typically, this method works within a certain range of parameters near an exchange stability bifurcation. This occurs when a one-site breather becomes unstable and a two-site breather does the opposite at a nearby point.

We have looked for mobile breathers in our system both with a hard $\phi^{4}$ potential and with a Morse potential, but we have only had success with Morse potential and "ferromagnetic" interaction, i.e., $\theta_{t w}>90^{\circ}$. In this particular case, we found a similar situation to a stability exchange and we were able to move the breather perturbing it with the unstable localized mode of the two-site breather. This is an interesting result because this configuration is equivalent to a chain of antiparallel dipoles twisted $\pi-\theta_{t w}<\pi / 2$. In fact, we can only expect parallel dipoles in synthetic DNA.

A useful concept for describing the breather movement is its effective mass. If the norm of the perturbation velocity is $\lambda$, the kinetic energy added to the breather by the perturbation is $E=\lambda^{2} / 2$. The resulting translational velocity of the breather, $v$, is found to be proportional to $\lambda[10]$. Thus, moving breathers can be considered as a quasiparticle with a mass of $m^{*}$, which can be defined through the relation $m^{*} v^{2} / 2=\lambda^{2} / 2$.

We have studied the dependence of the effective mass, $m^{*}$, with the coupling $J$. Figure 5 shows the result for antiparallel dipoles $\left(\theta_{t w}=180^{\circ}\right)$. Two different behaviors were obtained depending on the initial conditions. If we perturb the two-site breather, we observe that its effective mass increases monotonically with the coupling (full squares in Fig. 5). This reflects the fact that the two-site breather becomes stable with increasing coupling. But if a static one-site breather is chosen as the initial configuration, a minimum of $m^{*}$ appears showing the existence of an optimal value of the coupling to move this breather (see blank squares in Fig. 5). We believe that this minimum expresses a balance between the two opposite effects produced by an increase of the coupling on the stability of the one-site and two-site breathers. Similar results are obtained for other values $\theta_{t w}>\pi / 2$. 


\section{CONCLUSIONS}

We have considered a system of oscillating dipoles with helicoidal structure in order to study the effect of helicity on the existence and properties of breathers. This study is motivated by the helicoidal structure of DNA, and the fact that it can be described by a reduced dynamics where the only degrees of freedom are the stretchings of the hydrogen bonds between base pairs, which have a finite dipole moment. In our model, the helicity produces a narrowing of the phonon band, and an enlargement of the range of existence and stability of the breathers, although this effect is small for a typical helicoidal structure of DNA.
The effect of the orientation of the dipole moments, i.e., if the twisting angle is greater or not than $90^{\circ}$, is however considerably higher. In particular, we have only found mobile breathers with a Morse potential and $\theta_{t w}>\pi / 2$. Understanding the necessary conditions to move a breather is still an open question today.

\section{ACKNOWLEDGMENTS}

This work was supported by the European Union under the RTN project, LOCNET, HPRN-CT-1999-00163. We acknowledge Jesús Cuevas for his useful comments.
[1] M. Peyrard and A.R. Bishop, Phys. Rev. Lett. 62, 2755 (1989).

[2] M. Salerno and Yu. Kivshar, Phys. Lett. A 193, 263 (1994).

[3] P.L. Christiansen, YB. Gaididei, and S.F. Mingaleev, J. Phys.: Condens. Matter 13, 1181 (2001); Yu.B. Gaididei, S.F. Mingaleev, and P.L. Christiansen, Phys. Rev. E 62, R53 (2000); S.F. Mingaleev, P.L. Christiansen, Yu.B. Gaididei, M. Johansson, and K.Ф. Rasmussen, J. Biol. Phys. 25, 41 (1999).

[4] S.F. Mingaleev, YB. Gaididei, E. Majernikova, and S. Shpyrko, Phys. Rev. E 61, 4454 (2000).

[5] J.F.R. Archilla, P.L. Christiansen, S.F. Mingaleev, and Yu.B. Gaididei, J. Phys. A 34, 6363 (2001); J.F.R. Archilla, P.L. Christiansen, and Yu.B. Gaididei Phys. Rev. E 65, 16609 (2002).

[6] J. Cuevas, J. F. R. Archilla, Yu. B. Gaididei, and F. R. Romero,
Physica D (to be published).

[7] C. Calladine and H. Drew, Understanding DNA (Academic Press, London, 1992).

[8] M. Barbi, S. Cocco, and M. Peyrard, Phys. Lett. A 253, 358 (1999). M. Barbi, S. Cocco, M. Peyrard, and S. Ruffo, J. Mol. Biol. 24, 97 (1999); G. Gaeta, C. Reisss, M. Peyrard, and T. Dauxois, Riv. Nuovo Cimento 17, 1 (1994).

[9] R.S. MacKay and S. Aubry, Nonlinearity 7, 1623 (1994); S. Flach and C.R. Willis, Phys. Rep. 295, 181 (1998).

[10] Ding Chen, S. Aubry, and G.P. Tsironis, Phys. Rev. Lett. 77, 4776 (1996); S. Aubry and T. Cretegny, Physica D 119, 34 (1998).

[11] J.L. Marin and S. Aubry, Nonlinearity 9, 1501 (1996).

[12] S. Aubry, Physica D 103, 201 (1997). 